\title{
EPILEPSIA E DOENÇA DE CHAGAS CRONICA
}

\author{
EDYMAR JARDIM * \\ OSVALDO M. TAKAYANAGUI **
}

A síndrome epiléptica em pacientes chagásicos agudos ou crônicos tem sido assinalada por vários autores, mais frequentemente nos casos agudos da doença. Assim, foi referida por Austregesilo ${ }^{2}$, Cardoso ${ }^{6}$, Chagas 7, Evandro Chagas 10, Collares-Moreira 11, Elejalde 13, Mazza 24, entre outros. Entretanto, parece-nos que o estudo detalhado das manifestações epilépticas, na vigância da doença de Chagas crônica, da semiologia correspondente e dos resultados terapêuticos, não tenha sido realizado. A bibliografia consultada limita-se ao registro das eventuais manifestações convulsivas, $s \in m$ tecer comentários de natureza diagnóstica ou terapêutica.

A agressão ao sistema nervoso central na doença de Chagas aguda ou crônica está comprovada em estudos morfológicos e clínicos realizados no âmbito experimental e humano, considerando-se para tanto 03 trabalhos de Armbrust-Figueiredo e Jardim ${ }^{1}$, Britto-Costa ${ }^{4}$, Britto-Costa e Gallina 5, Jar$\operatorname{dim} 16,17,18,19,20$, Menezes 27, Schwartzburd e Köberle 30, Vichi 31, Reis 29, Lomonaco ${ }^{23}$, Vieira ${ }^{32}$, Vieira e col. ${ }^{33}$, Iazigi ${ }^{15}$, Kimachi ${ }^{22}$.

Alguns destes autores demonstraram em estudos histopatológicos, graus variáveis de destruição neuronal $\mathrm{em}$ vários níveis do sistema nervoso central. Ulteriormente, pesquisas clínicas baseadas nas alterações morfológicas encontradas, caracterizaram através de testes funcionais, perturbações da homeostase em geral, tais como as disfunções da sudação, da potassemia, da iodoproteinemia, do cortisol plasmático, das provas de tolerância a glicose. Entretanto, tais funções são mantidas por contingantes neuronais integrantes de centros nervosos específicos, particularmente localizados ou relacionados intimamente com a região hipotalâmica. Qual seria a resposta a um estímulo agindo sobre um encéfalo chagásico, que envolvesse em sua elaboração, não apenas grupamentos neuronais nucleares, mas grandes contingentes de células nervosas, como no caso das epilepsias? Eis aqui a razão para esta pesquisa. Este trabalho pretende comparar dois grupos de epilépticos, um dos quais com doença de Chagas crônica. Não desejamos estabelecer relação casual entre a doença e a sindrome epiléptica. sendo nosso objetivo verificar, se pelo fato da doença de Chagas comprometer efetivamente o sistema nervoso central, as manifestações epilépticas, a semiologia neurológica e a resposta terapêutica, serão diferentes num e noutro grupo.

Trabalho do Departamento de Neuropsiquiatria e Psicologia Médica da Faculdade de Medicina de Ribeirão Preto da Universidade de São Paulo: * Professor Adjunto de Neurologia; ** Pós-Graduando de Neurologia. 


\section{MATERIAL E METODOS}

Foram estudados 167 pacientes do Ambbulatório de Neurologia do Hospital das Clínicas cla FMRP, todos com o diagnóstico de síndrome epiléptica. Dəstes, 44 eram chagásicos crônicos, considerando-se para tanto os antecedentes epidemiológicos e a reação de Machado-Guerreiro praticada no sangue. Não foi realizada seleção prévia dos pacientes.

o estudo permitiu coletar informações referentes à proce ¿ência, sexo, idade, época de incidência das manifestações epilépticas aos tipos de crises, ao exame neurológico, ao exame do liquido cefalorraqueano, ao eletrencefalograma e aos resultados da terapêutica anticonvulsivante.

\section{RESULTADOS}

A maior parte dos pacientes examina $20 s$ em ambos os grupos eram procedentes do Ribeirão Preto (SP) ou imediações (95\%); os demais, oriundos de várias localidades do Estado de Minas Gerais (5\%). A reação de Machado-Guerreiro praticada no sangue dos mesmos, está representada em seus valores na tabela 1. A distribuição da casuística quanto aos sexos mostrou-se semelhante, sendo especificamente no grupo chagásico de 24 pacientes do sexo masculino e 20 co feminino. As idades no grupo chagásico variaram de 1 ano e 8 meses a 62 anos; no grapo controle de 2 anos e 7 meses a 66 anos.

Relativamente à época de aparecimento das manifestações epilépticas evidenciou-se que estas ocorreram mais tardiamente no grupo chagásico, aparecendo após os 25 anos de idade em $75 \%$ dos casos. No grupo controle, ao contrário, o inicio das manifestações epilépticas ocorreram em $70 \%$ dos casos antes dos 25 anos.

\begin{tabular}{cc}
\hline Titulo & Número de casos \\
\hline maior q:ee 1,5 & 1 \\
maior que 1,7 & 2 \\
maior que 1,8 & 1 \\
maior que 2,1 & 4 \\
maior que 2,2 & 2 \\
maior que 2,3 & 6 \\
maior que 2,6 & 8 \\
maior que 2,8 & 1 \\
maior que 3,0 & 19 \\
\hline Tabela 1 - Resultados da reacão de Machado-Guer- \\
reiro praticada no sangue de 44 pacientes \\
chagdsicos com sindromes epilépticas.
\end{tabular}




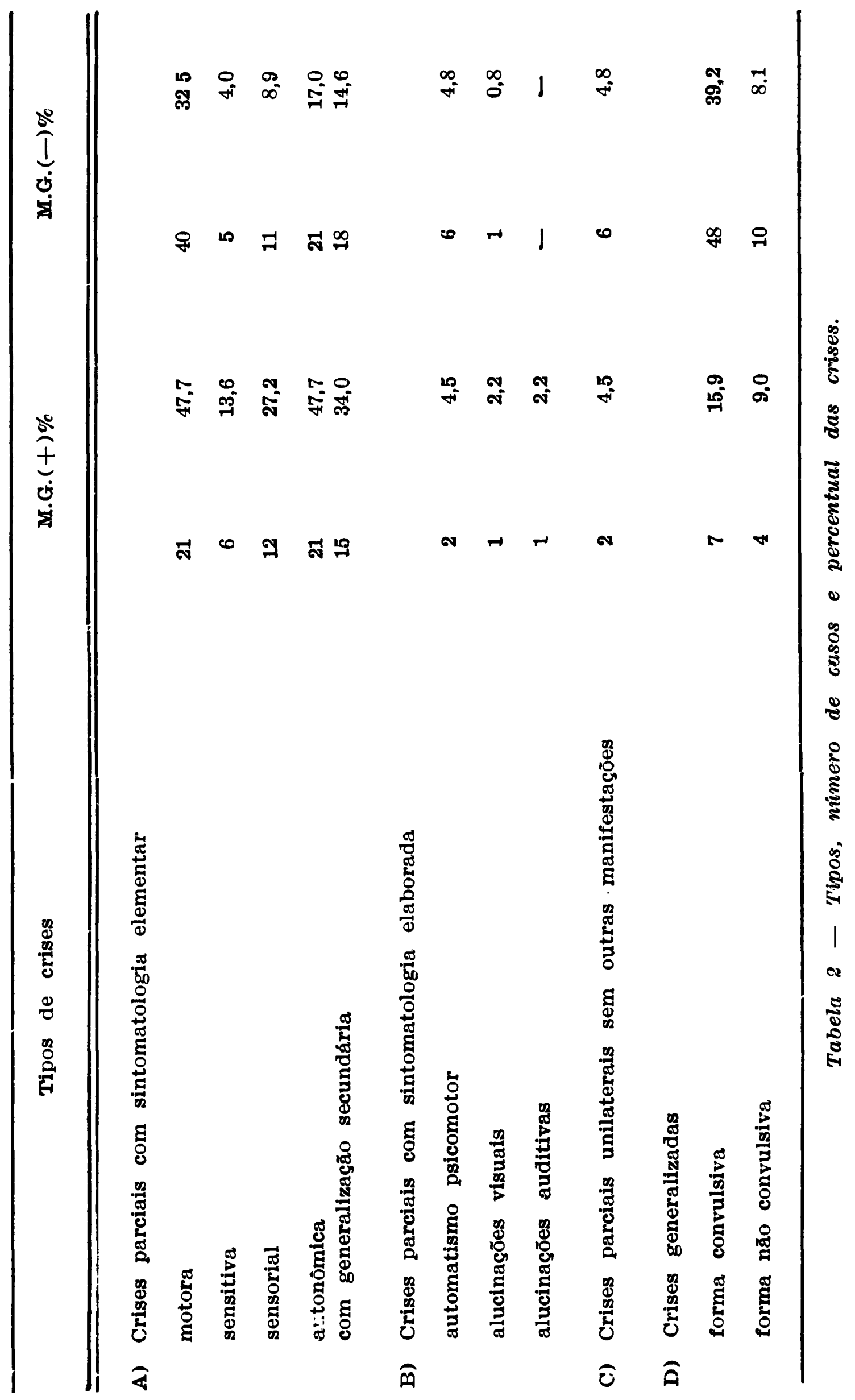


Quanto aos tipos das crises epilépticas (Tabela 2), encontramos grande predominância no grupo chagásico, de crises parciais com sintomatologia elementar de tipo autonômico. As crises parciais com sintomatologia elaborada, foram encontradas em percentagens praticamente iguais para ambos os grupos. As crises gcneralizadas foram assinaladas com maior frequência no gr. po não chagásico.

O exame neurológico (Tabela 3) mostrou que no grupo chagásico as alterações semiológicas foram encontradas mais vezes. Estas alterações, com algumas exceções, foram na maior parte dos pacientes examinados, isoladas, não constituindo uma síndrome neurológica perfeitamente caracterizada. Fato semelhante registramos em relação ao exame de liquido cefalorraqueano (Tabela 4) onde a maior incidência de alterações no grupo chagásico, constituiu-se de variações de um ou outro elemento de rotina laboratorial, sem contudo, definir uma síndrome liquórica. As reaçóes de MachadoGuerreiro efetuadas no líquido cefalorraqueano foram sempre negativas.

Foram realizados exames eletrencefalográficos em 15 epilépticos chagásicos crônicos, sendo os traçados comparados com um grupo controle não chagásico. Verificou-se neste estudo, que na maior parte dos pacientes do grupo chagásico há evidentes diferenças na organização da atividade de fundo com maior incidência de rítmos rápidos difusos, e menor diferenciação postero-anterior dos rítmos cerebrais. Não foram encontrados elementos expressivos quanto à ocorrência de elevação de indice teta. nem características suficientes para comprovar diferenças na incidència de assismetria de rítmos posteriores ou de difusão anterior de rítmo alfa. Relativamente as atividades paroxísticas, foi observada maior incidência de ondas lentas localizadas (frequência delta e teta) nos pacientes chagásicos.

Quanto aos resultados da terapêutica anticonvulsivante utilizada (Tabela 5), baseada no emprego de hidantoinatos, primidona, benzodiazepínicos e barbitúricos, foi possível ccnstatar-se que as respostas foram muito melhores no grupo controle qiie no chagásico.

\section{COMENTARIOS}

O exame de nossa casuística não nos deu a impressão de que houvessem diferenças nas manifestações de natureza epiléptica dependendo do sexo ou idade. cios pacientes.

Verificamos, entretanto, que os inícios das manifestações epilépticas nos chagásicos ocorreu mais tardiamente quando em comparação ao grupo controle. Aliás, é fato amplamente documentado que muitas das alterações patológicas decorrentes da moléstia de Chagas crônica, tais como o megacolo, o megaesôfago e a cardiopatia ocorrem depois de uma evolução relativamente longa da doença. Parece-nos que o mesmo ocorre com a epilepsia. As crises parciais foram predominantes no grupo chagásico, fato assinalado por Medrado-Faria e col.26, em extensa casuística. Na classificação das crises parciais, tiveram grande evidência as crises com sintomatologia elementar de tipo autonômico. $E$ interessante lembrar que grande parte das manifestações da doença de Chagas crônica são de natureza autonômica, particularmente aquelas ligadas à regulação da homeostase, como demonstraram Reis ${ }^{29}$, Lomonaco ${ }^{23}$, Jardim ${ }^{17}$, Vieira e col. ${ }^{33}$, Iazigi e col.15, Kimachi e col.22. 
M.G.(+) M.G.(一)

1. Consciência

estado confusional

2. Força muscular

hemiparesia

5

hemiplegia

monoparesia crural

1

3. Tono muscular

hipotonia global

hipertonia piramidal dimidiada

mioclonias

1

1

4. Trofismo

hipotrofia global

hemihipotrofia facial

$\begin{array}{ll}2 & 1 \\ - & 1 \\ - & 1 \\ 2 & - \\ - & 2\end{array}$

5. Coordenação

hemiataxia

ataxia giobal

- 1

$-1$

6. Equilibrio

sinal de Romberg

1

7. Sensibilidade

hemihipoestesia superficial dimidiaju

hemihipoestesia sup. e prof. dimidiada

8. Reflexos profundos

hiporreflexia global

hiperrerlexia dimidiada

huperrerlexia e sinais patológicos

4

32

9. Reflexos superficiais

hiporreflexia global

10. Nervos cranianos

10 anosmia bilateral

1

2. amaurose unilateral

29 atrofia papiıar

29 corioretınite

1

30 anısocoria

$5^{\circ}$ hemihipoestesia facial

70 paresia facial central

80 hipoac sia unilateral

129 hemihipotrofia lingual 


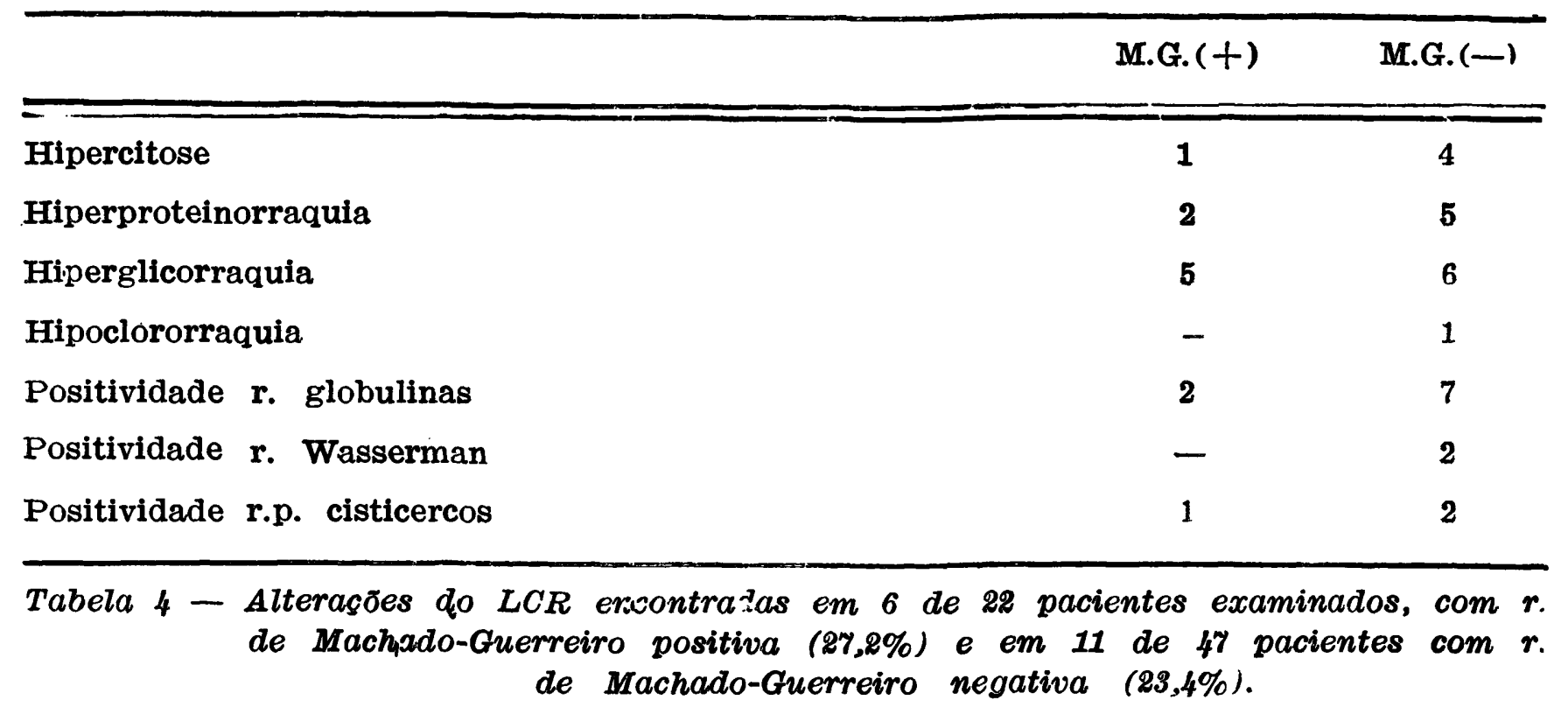

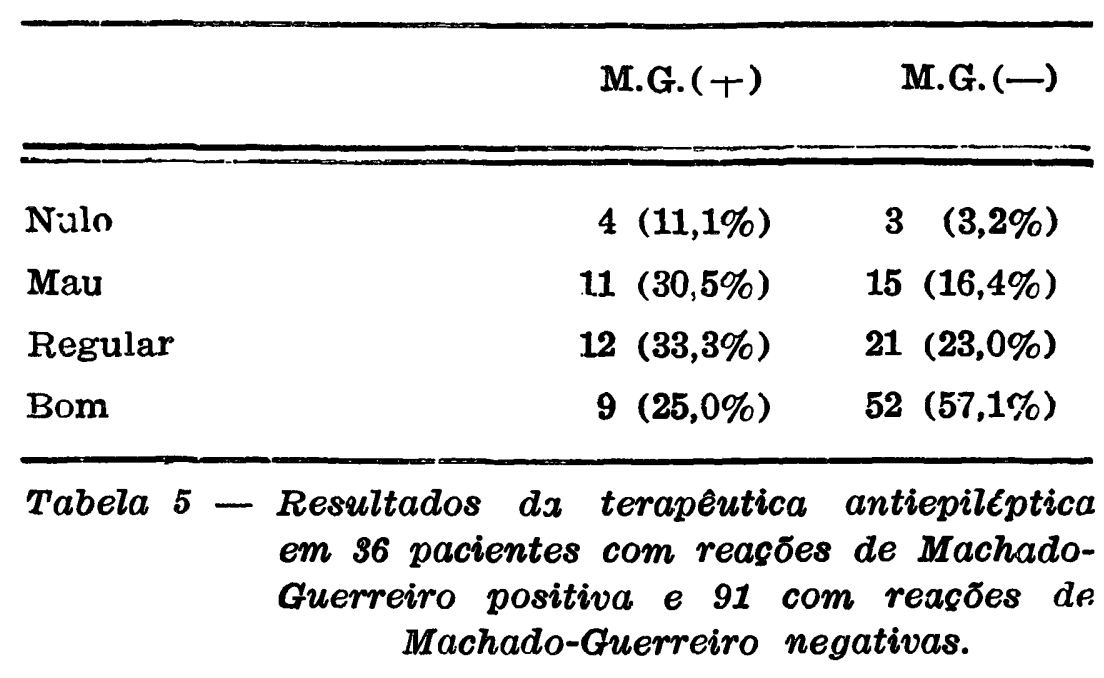

Os exames neurológicos mostraram que no grupo chagásico as alterações semiológ:cas isoladas foram moderadamente mais frequentes, como já assinalamos anteriormente 20. As síndromes neurológicas perfeitamente caracterizadas, constituiram-se de: cinco casns de hemiparesia (dois com hemihipoestesia superficial e profunda), em que os pacientes tinham antecedentes sugestivos de acidentes vasculares cerebrais de tipo isquêmico; um caso de anosmia e amaurose unilateral subsequentes a trauma cranioencefálico; um caso de monoparesia crural de etiologia não estabelecida. Os demais elementos semiológicos registrados não caracterizavam síndromes neurológicas definidas.

Os resultados encontrados nos exames do líquido cefalorraqueano (LCR), não nos permitiram caracterizar qualquer síndrome orgânica neurológica. Apesar de Chagas $\tau$ ter registrado em suas observações iniciais sobre a doença, alterações no LCR de chagásicos crônicos, a literatura consultada $3,21,28$, com exceção dos trabalhos de Vilela e Bicalho ${ }^{34}$ e de Colares-Moreira 11 não confirma aqueles achados. Todavia, recentemente, Marquez e col.25, examinando o LCR de 43 chagásicos crônicos, demonstraram que utilizando-se a concentração do LCR 
pelo método de Meies, é possivel demonstrar-se percentagens elevadas de anticorpos contra neurônios, endocárdio, vasos, interstício e nervos.

As referências bibliográficas relativas à exames eletrencefalográficos realizacios em pacientes chagásicos crônicos são escassas. Käfer e col. 21 estudaram 30 pacientes com reações de Machado-Guerreiro positiva no sangue, dois terços dos quais tinham cardiopatias, encontrados $50 \%$ de traçados eletrencefalográficos que consideraram como "anormais", Di Lascio 12, analisando o traçado de 15 pacientes chagásicos crônicos com patologias digestivas, sem qualquer evidência de comprometimento do sistema nervoso, concluiu pela normalidade da eletrogênese cerebral. Girardelli ${ }^{14}$ examinando 74 pacientes jovens entre as idades de 4 a 19 anos, comprovadamente chagásicos através de elementos epidemiológicos e sorológicos encontrou $70,3 \%$ de traçados patológicos. O estudo comparativo com casuística de igual número e idade e reações sorológica: negativas, revelou apenas $10,8 \%$ de traçados considerados "anormais". Diante das alterações eletrencefalográficas que encontramos em nossos pacientes, tivemos a impressão de que as mesmas, possam representar consequências de um processo orgânico cerebral de natureza difusa.

Quanto à terapêutica antiepiléptica, os chagásicos exigiram doses medicamentosas substancialmente maiores que os pacientes do grupo controle, com resultados, bastante inferiores no que se refere ao combate às suas crises Esta condição de dificuldade na obtenção de bons resultados no controle das crises nos chagásicos, parece-nos compatível com a idéia de que a moléstia de Chagas em nível de sistema nervoso central, seja representada por processo orgânico cerebral difuso, como sugeriram os exames realizados.

\section{RESUMO}

A sindrome epiléptica em chagásicos crônicos, foi referida raramente na literatura especializazda, não tendo sido feito até o momento, um estudo detalhado das suas manifestações.

Partindo-se da premissa de que a moléstia de Chagas tem por substrato anatômico uma destruição neuronal, procurou-se comparar dois grupos de epilépticos, um dos quais com moléstia de Chagas crônica. Foram estudados 167 pacientes epilépticos, dos quais 44 eram comprovadamente chagásicos. $\mathrm{O}$ estudo permitiu coletar dados referentes à procedência dos pacientes, resultado sorológico, sexo, idade, época de incidência das manifestações epilépticas, elementos dos exames neurológicos, do líquido cefalorraqueano, eletrencefalográfico e os resultados da terapêutica anticonvulsivante.

Como resultados principais destacamos o inicio tardio da epilepsia nos chagásicos, e o predomínio acentuado das crises parciais com sintomatologia elementar de tipo autonômico. O exame neurológico e o do líquido cefalorraqueano, apesar de apresentarem percentualmente nos seus resultados, taxas moderadamente mais elevadas na incidência de alterações, não caracterizaram 
sindromes neurológicas bem definidas. $O$ exame eletrencefalográfico, revelou alterações sugestivas de comprometimento orgânico cerebral difuso*.

A terapêutica anticonvulsivante, baseada na utilização de hidantoinatos, oarbitúricos, primidona e benzodiazepínicos, mostrou que o controle das crises foi mais difícil nos chagásicos, exigindo maiores quantidades de medicação, com resultados menos satisfatórios.

\section{SUMMARY}

\section{Epilepsy and chronic Chagas'disease.}

The epileptic syndrome in chronic Chagas'disease is rarely reported in neurological literature. At the present time many papers have demonstrated that histopathological basis of Chagas'disease is a neuronal destruction. The author: studied 161 epileptic patıenis; 44 out of them had a chronic form of the disease. It was made a compariso.l of semiologic data between the two groups, and also the evaluation of the therapeutic results with anticonvulsant drugs. The chagasic patients had the onset of epileptic seizures later than the control group. witil great predominance of partial seizures of autonomic type. The neurologic examination and cerebrospinal fluid test revelead moderate rates of disturbances, but not sufficient to characterize a neurologic syndrome. The EEG study was performed in 15 of the 44 cases and revealed a suggestive pattern of a diffuse cerebral damage in half of patients. Anticonvulsant therapy based on use of phenylhidantoine, barbituric acid derivates, primidone and benzodiazepines, showed that control of epileptic seizures in Chagas'diseases is more difficult and requires greater quantities of drugs than in the control group.

\section{REFERENCIAS}

1. ARMBRUST-FIGUEIREDO, J. \& JARDIM, E. - The diagnosis of chronic nervous iorm of Chagas disease. $X$ International Congres of ivuuroiogy, B:trce.ona, Espanha. bixcerpta Médica 296:11, 1973.

2. AUStregesilo, A. - Formes nerveuses de la maladie ie Chagas. Rev. Neurol. (Paris) 1:1, 192\%.

3. BASSO, G. \& BASSO, R. - Est..dios de enfermos del Asilo de Mendoza con reacion de Machado positiva. An. IX Reunion Soc. Argent. Pat. Reg. Mendozza, 1936.

4. BRITTO-COSTA, R. - Alterações hipotalâmicas na fase crônica da moléstia de Chagas experimental. Rev. Inșt. Med. Troł. São Paulo 13:336, 1971.

5. BRITTO-COSTA, R. \& GALLINA, R. A. - Hipotálamo anterior na moléstia de Chugas humana. Rev. Inst. Med. 'Trup. São Paulo 13:92, 1971.

* Nota dos autores - Agradecemos ao Prof. Michel Pierre Lison a colaboração na análise dos traçados eletrencefalográficos. 
6. CARDoso, R. A. A. - Lesões do sistema nervoso central em quatro casos infantis agudos da doença de Chagas. Bol. Inst. Puer. Univ. Brasil 17:101, 1960.

7. Chagas, C. - Nova entidade mórbida do homem. Resimo geral de estudos etiológicos e clínicos. Mem. Inst. O. Cruz (Rio de Janeiro) 3:219, 1911.

8. CHAGAS, C. - Les formes nerveuses d'une neuvelle trypanosomiase. Nouv. Iconogr. Salpetrière 26:1, 1913.

9. CHAGAS, C. - American trypanosomiasis: study of the parasite and of the transmitting insect. Chicago Med. Rec. 43:609, 1921.

10. CHAGAS, E. - Súmula dos conhecimentos atuais sobre a tripanosomíase americana. Mem. Inst. O. Cruz (Rio de Janeiro) 30:387, 1940.

11. COLLARES-MOREIRA, J. V. - A forma nervosa da moléstia de Chagas. Tese. Fac. Med. Rio de Janeiro, 1925.

12. DI LASCIO, A. - E. E. G. em portadores de doença de Chagas (forma crôniça). Neurobiologia (Recife) 28:99, 1965.

13. ELeJALDE, P. - Lesões cerebrais na doença de Chagas aguda. An. II Congr. Lat. Amer. Anat. Pat. São Paulo, 1958.

14. GIRARDELLI, M. A. - Electroencefalografia y enfermedad de Chagas cronica. Bol. Chileno Parasitol. 24:32, 1969.

15. IAZIGI, N.; LOMONACO, D. A.; VERISSIMO, J. M. T. \& OLIVEIRA, H. L. Função tireoidiana de ratos com infeç̧ão chagásica crônica: influência do teor de iodo na dieta. Rev. Ass. Med. Brasil (São Paulo) 17:227, 1971.

16. JARDIM, E. - Alterações quantitativas das célilas de Purkinje na moléstia de Chagas experimental no camunđongo. Arq. Neuro-Psiquiat. (São Paulo) 25:199. 1967.

17. JARDIM, E. - Sudorese em pacientes com moléstia de Chagas crônica. Arq. NeuroPsiquiat. (São Paulo) 25:214, 1967.

18. JARDIM, E. - Moléstia de Chagas aguda experimental: parasitismo do hipotálamo. Arq. Neuro-Psiquiat. (São Paulo) 29:190, 1971.

19. JARDIM, E. - Moléstia de Chagas experimental no rato: parasitismo do núcleo do III par craniano. Rev. Inst. Med. Trop. São Paulo 13:405, 1971.

20. JARDIM, E. \& ARMBRUST-FIGUEIREDO. J. - Estudo neurológico e do líquido cefalorraqueano em pacientes com a forma crônica da moléstia de Chagas. Arq. Neuro-Psiquiat. (São Paulo) 30:332, 1972.

21. KAFER, J. P.; POCH, G. F.; MONTEVERDE, D. A.; FERNANDEZ-BLANCO, E. \& TARSIA, R. - Las manifestaciones neurológicas en la forma cronica de la enfermedad de Chagas. Rev. Neurol. Buenos Aires 9:199, 1961.

22. KIMACHI, R.; LOMONACO, D. A. \& VERISSIMO, J. M. T. - Exploração funcional do eixo hipotálamo-adenohipófise-cortex adrenal na forma crônica da moléstia de Chagas. Rev. Ass. Med. Brasil (São Paulo) 20:57, 1974.

23. LOMONACO, D. A. - Estudo da função tireoidiana na forma crônica da moléstia de Chagas. Tese de Docência-Livre, Faculdado de Medicina de Ribeirão Preto, Universidade de Sťo Paulo, 1962. 
24. MAZZA, S. - Formas meningoencefálicas primitivas y secundarias de la enfermidad de Chagas. Mission de Estudios de Patologia Regional Argentina 60:3, 1942.

25. MARQUEZ, J. O.; JARDIM, E.; LOPES, E. R. \& SANTOS, R. R. - Imunopatologia do líquido cefalorraqueano na doença de Chagas. Rev. Neurol. Argent. 3:448, 1977.

26. MEDRADO-FARIA, M. A.; MARQUES-ASSIS, L.; SILVA, G. R. \& CAMARGO. M. E. - Infecção chagásica crônica e síndrome epiléptica. Rev. Inst. Med. Trop. São Paulo 18:173, 1976.

27. MENEZES, H. - Moléstia de Chagas experimental: lesões do sistema nervoso central na fase aguda. Rev. Bras. Med. (Rio de Janeiro) 21:21, 1964.

28. PEDREIRA DE FREITAS, J. L. \& MENDES, R. T. - Investigações sorológicas na forma nervosa crônica da moléstia de Chagas entre pacientes internados em hospital psiquiátrico * Rev. Paulista Med. 46:123, 1955.

29. REIS, L. C. F.; OLIVEIRA, H. L. \& VIEIRA, C. B. - Curvas glicêmicas anormais observadas em pacientes com a forma crônica da moléstia de Chagas. Rev. Goiana de Med. 1960.

30. SCHWARTZBURD, H. \& KÖBERLE, F. - Chagas Myelopathie. Zschr. F. Tropenmej. n. Parasit. 10:309, 1959.

31. VICHI, F. L. - Destruição de neurônios motores na medula espinal de ratos na fase ag.da da moléstia de Chagas. Rev. Inst. Mel. Trop. São Paulo 6:150, 1964.

32. VIEIRA, C. B. - Hipersudação provocada pela pilocarpina na moléstia de Chagas crônica. Hospital (Rio de Janeiro) 64:57, 1963.

33. VIEIRA, C. B.; MAZZONCINI, M. \& LOMONACO, D. A. - Variações da potassemia na forma crônica da moléstia de Chagas. Rev. Paulista Med. 66:239, 1965.

34. VILLELA, E. \& BICALHO, J. C. - As pesquisas de laboratório na moléstia de Chagas. Mem. Inst. O. Cruz (Rio de Janeiro) 16:13, 1923.

Departamento de Neuropsiquiatria e Psicologia Médica (Disciplina de Neurologia) - Fraculdade de Medicina de Ribeirão Preto - Av. 9 de Julho 980 - 14100 Ribeirão Preto, SP - Brasit. 